Research Article

\title{
A Structural Analysis Method for Cable-Beam Composite Structure
}

\author{
Guoqiang You \\ Xi'an Fanyi University, Taiyigong Street, Xi'an, China \\ Correspondence should be addressed to Guoqiang You; simonyouyou@163.com
}

Received 28 January 2020; Revised 3 May 2020; Accepted 9 May 2020; Published 1 June 2020

Academic Editor: Francesco Cannizzaro

Copyright (c) 2020 Guoqiang You. This is an open access article distributed under the Creative Commons Attribution License, which permits unrestricted use, distribution, and reproduction in any medium, provided the original work is properly cited.

\begin{abstract}
A structural analysis method is proposed in this paper for cable-beam composite structure with a large number of cable elements; it can improve computational efficiency and accuracy. Firstly, we use a structural division method to divide the whole cable-beam structure into inner cablenet system and edge cablenet-beam system. Because inner cablenet is a pure cablenet structure with given shape and topology, balance matrix analysis method is used to help obtain the best pretension distribution of it with the evenness as objective. For edge cablenet-beam system, with obtaining equilibrium forces of inner cablenet's pretensions as goal, an iterative pretension losses compensation calculation combined with nonlinear finite element method and balance matrix analysis method is used for edge cablenet to adjust its pretension distribution and make the whole cablenet system keep its preset ideal shape under influence of beams' deformations. Because edge cablenet-beam system has a lot less cable elements than overall structure, its nonlinear finite element calculations are much easier and faster than those for the whole cable-beam composite structure. In order to verify the above method's validity, it is used for a spaceborne cablenet deployable antenna example, and the computed results indicate that the method is feasible.
\end{abstract}

\section{Introduction}

In engineering practice, cablenets in cable-beam composite structures are usually tension systems with predetermined topologies and shapes. The structural analyses for these cablenet systems are aimed at obtaining a set of reasonable cable pretensions to make sure the whole cable-beam composite structure has enough strength and stiffness along with an ideal shape [1].

Nonlinear finite element method is often used for analyzing structural properties of cable-beam composite structures. For cable-beam composite structure with strict demand for shape precision, this method is commonly combined with optimization algorithms or inverse iteration method to iteratively control nodal deformations to reduce structure's shape errors and ensure an accurate structural shape. Papers [2-4] use nonlinear finite element method and optimization methods to optimize the structural parameters of cable-beam composite structures, and, by calculating with cable elements' cross-sectional areas or pretensions as variables and structural shape precision as goal, they obtain the optimal cable pretension distribution and a satisfactory shape precision. Papers [5-7] analyze cable-beam composite structures by combining form finding method and inverse iteration method. Because inverse iteration method can iteratively adjust nodal positions to meet structure's shape precision demand, this comprehensive method can make sure the cablenet system of the structure always has an ideal shape precision and desired pretension distribution.

Although the above methods can be used for analyzing and designing cable-beam composite structures, the structural interactions between beam system and cablenet system are not considered in these methods, and, for large-scale cable-beam composite structures with complex topology and a large number of cable elements, the problems such as divergence of nonlinear finite element calculation and inefficiency of optimization are also hard to prevent. In order to better understand and solve these problems and make sure the cablenet system has an ideal shape precision and pretension distribution under influences of beams' 
deformations, this paper proposes a division method for cable-beam composite structures, and, by calculating substructures in different ways, the method can efficiently and accurately analyze and design complex cable-beam composite structures.

In this method, the cable-beam composite structure is divided into two parts, inner cablenet system and edge cablebeam system. Inner cablenet system is made up of cable elements with given positions and topology, and, for this part, balance matrix analysis method can be used to efficiently analyze this type of cablenet system $[8,9]$. The other part, edge cable-beam system, also is a type of cable-beam composite structure, but this substructure has a lot fewer cable elements than original cable-beam structure. Because balance matrix analysis method cannot add effects of beams' deformations to structural analysis of cablenet system, if still using this method to analyze edge cable-beam system , the results of calculation will be rough and imprecise. In order to reflect interactions of beam system and cablenet system, balance matrix analysis method and nonlinear finite element method are combined in this paper, and their respective advantages can both be used for better analyzing edge cablebeam system to obtain more accurate results.

\section{The Division Method for Cable-Beam Composite Structure}

2.1. Basic Description for the Division Method. In this paper, cable-beam composite structure is first divided into two parts, and then these two parts are calculated with various best ways. The purpose of this division method is meant to greatly reduce computation cost and improve results' precision.

Means of the division method for cable-beam composite structures are as follows. Firstly, divide the cablenet system of cable-beam composite structure (shown as Figure 1) into inner cablenet system and edge cable-beam system, and set all joints connecting inner cablenet with edge cablenet to fixed nodes. Secondly, analyze and optimize inner cablenet to get its best pretension distribution. Thirdly, for the edge cable-beam structure which just has a simple cablenet topology with much fewer cable elements, iteratively adjust pretensions of edge cablenet to make them match with inner cablenet pretensions at cable joints between these two parts. Fourthly, put the optimal pretensions of inner cablenet and edge cablenet into original cable-beam composite structure model, and these pretensions will make sure the whole structure keeps an ideal shape precision. Because nodal forces of inner cablenet and edge cablenet at their connection joints can ensure an equilibrium force system, all cable pretensions of cable-beam composite structure will be stable, and the whole cablenet system can keep its ideal structural shape and preset pretension distribution.

When the above method is used to analyze cable-beam composite structure, the object of pretension adjustment is only the edge cablenet system with much fewer cable elements, and the object of nonlinear finite element calculation is just the simpler edge cable-beam structure, so this method can greatly reduce cost of computation.
2.2. Principles of the Division. Based on nonlinear finite element equations, the principles of the division method are further described here in theory.

The cable-beam composite structure shown in Figure 1 is also used as an example to illustrate the points. For more easily writing nonlinear finite element equations, the twenty-four fixed cable nodes in inner cablenet are classified as node-set $a$, and the other forty-five cable nodes are classified as node-set $b$. For edge cable-beam structure, its twenty-four fixed cable nodes are classified as node-set $a^{\prime}$, the four fixed nodes in beam elements are classified as node-set $c$, and the other twenty-four beam nodes are classified as node-set $d$. When denoting inner cablenet structure as S1 and edge cable-beam structure as S2, the relationships between nodes in S1 and S2 and the above node-sets can be written as

$$
\left\{\begin{array}{l}
N^{S 1}=\left\{N^{a}, N^{b}\right\}, \\
N^{S 2}=\left\{N^{a^{\prime}}, N^{c}, N^{d}\right\} .
\end{array}\right.
$$

Node-sets $a$ and $a^{\prime}$ have the same nodes, so, for these two sets' nodes with the same positions, their coordinate relations can be written as

$$
\left\{\begin{array}{l}
x^{a}=x^{a^{\prime}}, \\
y^{a}=y^{a^{\prime}}, \\
z^{a}=z^{a^{\prime}} .
\end{array}\right.
$$

Symbols $x, y$, and $z$ in equation (2) are node's three-axis values in global coordinate system.

By the above classification, nonlinear finite element equations of structures S1 and S2 cab be derived as follows.

First, assume that structure S1 is under the effects of prestress system $\sigma_{1}$, and its structural tangent stiffness matrix can be given as

$$
\mathbf{K}_{1}\left(\sigma_{1}\right)=\left[\begin{array}{ll}
\mathbf{k}_{1}^{\mathbf{a a}} & \mathbf{k}_{1}^{\mathbf{a b}} \\
\mathbf{k}_{1}^{\mathbf{b a}} & \mathbf{k}_{1}^{\mathbf{b b}}
\end{array}\right] .
$$

Then, nonlinear finite element equations of structure S1 can be further written as

$$
\left[\begin{array}{cc}
\mathbf{k}_{1}^{\mathbf{a a}} & \mathbf{k}_{1}^{\mathbf{a b}} \\
\mathbf{k}_{1}^{\mathbf{b a}} & \mathbf{k}_{1}^{\mathbf{b b}}
\end{array}\right]\left[\begin{array}{c}
\Delta \mathbf{u}_{1}^{\mathbf{a}} \\
\Delta \mathbf{u}_{1}^{\mathbf{b}}
\end{array}\right]=\left[\begin{array}{c}
\mathbf{R}_{1}^{\mathbf{a}} \\
\mathbf{R}_{1}^{\mathbf{b}}
\end{array}\right] .
$$

In equation (4), $\Delta \mathbf{u}_{1}^{\mathbf{a}}$ and $\Delta \mathbf{u}_{1}^{\mathbf{b}}$ are nodal displacement increments of node-sets $\mathbf{a}$ and $\mathbf{b} ; \mathbf{R}_{1}^{\mathbf{a}}$ and $\mathbf{R}_{1}^{\mathbf{b}}$ are unbalance forces of node-sets $\mathbf{a}$ and $\mathbf{b}$.

Considering DOF (degree of freedom) constraints from fixed nodes, equation (4) can be rewritten as

$$
\left[\begin{array}{cc}
1 & 0 \\
0 & \mathbf{k}_{1}^{\mathbf{b b}}
\end{array}\right]\left[\begin{array}{c}
0 \\
\Delta \mathbf{u}_{1}^{\mathbf{b}}
\end{array}\right]=\left[\begin{array}{c}
0 \\
\mathbf{R}_{1}^{\mathbf{b}}
\end{array}\right] .
$$

If $\mathrm{S} 1$ can be in its balance state under effects of prestress system $\sigma_{1}$, S1's $\Delta \mathbf{u}_{1}^{\mathbf{b}}$ and $\mathbf{R}_{1}^{\mathbf{b}}$ in this state must be zero.

Similarly, S2's structural tangent stiffness matrix under effects of prestress system $\sigma_{2}$ can be written as 


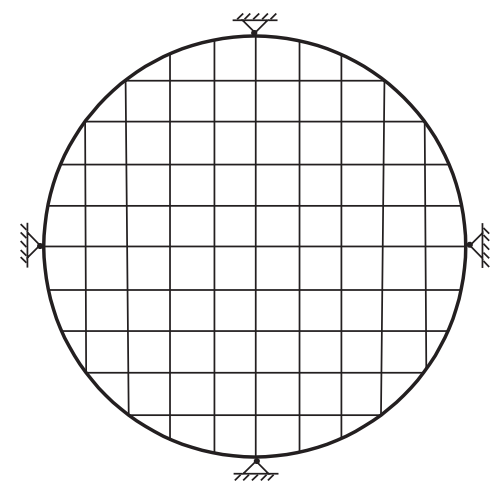

(a)

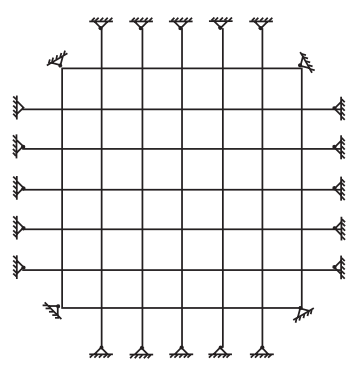

(b)

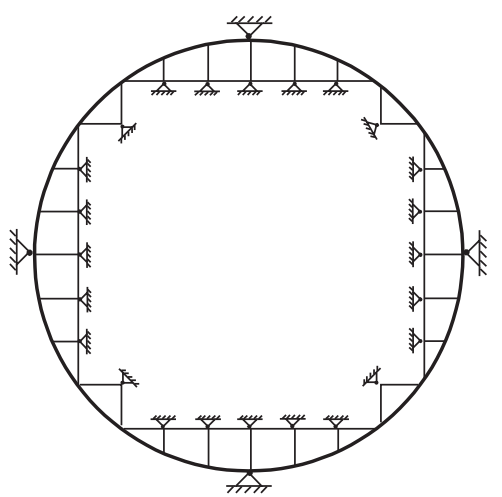

(c)

Figure 1: Division method for cable-beam composite structure. (a) A cable-beam composite structure. (b) Inner cablenet system after division. (c) Edge cable-beam system after division.

$$
\mathbf{K}_{2}\left(\sigma_{2}\right)=\left[\begin{array}{ccc}
\mathbf{k}_{2}^{\mathrm{a}^{\prime} \mathbf{a}} & \mathbf{k}_{2}^{\mathrm{a}^{\prime} \mathrm{c}} & \mathbf{k}_{2}^{\mathrm{a}^{\prime} \mathbf{d}} \\
\mathbf{k}_{2}^{\mathrm{ca}} & \mathbf{k}_{2}^{\mathrm{cc}} & \mathbf{k}_{2}^{\mathrm{cd}} \\
\mathbf{k}_{2}^{\mathrm{da}} & \mathbf{k}_{2}^{\mathrm{dc}} & \mathbf{k}_{2}^{\mathrm{dd}}
\end{array}\right]=\left[\begin{array}{ccc}
\mathbf{k}_{2}^{\mathrm{aa}} & \mathbf{k}_{2}^{\mathrm{ac}} & \mathbf{k}_{2}^{\mathrm{ad}} \\
\mathbf{k}_{2}^{\mathrm{ca}} & \mathbf{k}_{2}^{\mathrm{cc}} & \mathbf{k}_{2}^{\mathrm{cd}} \\
\mathbf{k}_{2}^{\mathrm{da}} & \mathbf{k}_{2}^{\mathrm{dc}} & \mathbf{k}_{2}^{\mathrm{dd}}
\end{array}\right] .
$$

Because node-sets $\mathbf{a}$ and $\mathbf{a}^{\prime}$ are made up of the same position nodes, symbol $\mathbf{a}^{\prime}$ in equation (6) can be replaced by $\mathbf{a}$.

Nonlinear finite element equations of structure S2 can be written as

$$
\left[\begin{array}{ccc}
\mathbf{k}_{2}^{\mathrm{aa}} & \mathbf{k}_{2}^{\mathrm{ac}} & \mathbf{k}_{2}^{\mathrm{ad}} \\
\mathbf{k}_{2}^{\mathrm{ca}} & \mathbf{k}_{2}^{\mathrm{cc}} & \mathbf{k}_{2}^{\mathrm{cd}} \\
\mathbf{k}_{2}^{\mathrm{da}} & \mathbf{k}_{2}^{\mathrm{dc}} & \mathbf{k}_{2}^{\mathrm{dd}}
\end{array}\right]\left[\begin{array}{c}
\Delta \mathbf{u}_{2}^{\mathrm{a}} \\
\Delta \mathbf{u}_{2}^{\mathrm{c}} \\
\Delta \mathbf{u}_{2}^{\mathrm{d}}
\end{array}\right]=\left[\begin{array}{c}
\mathbf{R}_{2}^{\mathrm{a}} \\
\mathbf{R}_{2}^{\mathrm{c}} \\
\mathbf{R}_{2}^{\mathrm{d}}
\end{array}\right]
$$

In equation (7), $\Delta \mathbf{u}_{2}^{\mathbf{a}}, \Delta \mathbf{u}_{2}^{\mathbf{c}}$, and $\Delta \mathbf{u}_{2}^{\mathbf{d}}$ are nodal displacement increments of node-sets $\mathbf{a}, \mathbf{c}$, and $\mathbf{d} . \mathbf{R}_{2}^{\mathbf{a}}, \mathbf{R}_{2}^{\mathbf{c}}$, and $\mathbf{R}_{2}^{\mathbf{d}}$ are unbalance forces of node-sets $\mathbf{a}, \mathbf{c}$, and $\mathbf{d}$.

Considering DOF constraints of fixed nodes, equation (7) can be rewritten as

$$
\left[\begin{array}{ccc}
\mathbf{1} & \mathbf{0} & \mathbf{0} \\
\mathbf{0} & \mathbf{1} & \mathbf{0} \\
\mathbf{0} & \mathbf{0} & \mathbf{k}_{2}^{\mathrm{dd}}
\end{array}\right]\left[\begin{array}{c}
0 \\
0 \\
\Delta \mathbf{u}_{2}^{\mathrm{d}}
\end{array}\right]=\left[\begin{array}{c}
0 \\
0 \\
\mathbf{R}_{2}^{\mathbf{d}}
\end{array}\right] .
$$

When S2 is in its balance state under effects of prestress system $\sigma_{2}$, S2's $\Delta \mathbf{u}_{2}^{\mathbf{d}}$ and $\mathbf{R}_{2}^{\mathbf{d}}$ in this state are all zero.

In view of the above nonlinear finite element equations of structures S1 and S2, we need next to study how to use them to compose original cable-beam composite structure's nonlinear finite element equations and derive conditioning that can make sure the composite structure keeps an ideal cablenet shape in the composing process.

Assume that S1 and S2 have been in their balance states under effects of prestress systems $\sigma_{1}$ and $\sigma_{2}$. Then release the DOF of node-sets a and $\mathbf{a}^{\prime}$, and join S1 and S2 together at these nodes to reform the whole cable-beam composite structure. Figures 2(a) and 2(b) show the inner cablenet structure and edge cable-beam structure released DOF constrains; Figure 2(c) shows the reformed cable-beam composite structure, and the white dots in this figure are the joints needed to connect edge cablenet and inner cablenet together.

Now, expand structural tangent stiffness matrices of S1 and S2, and join them together to get the whole structure's tangent stiffness matrix. The structural tangent stiffness matrix of S1 can be expanded in scale and rewritten as

$$
\mathbf{K}_{1}\left(\boldsymbol{\sigma}_{1}\right)=\left[\begin{array}{cccc}
\mathbf{k}_{1}^{\mathbf{a a}} & \mathbf{k}_{1}^{\mathbf{a b}} & \mathbf{0} & \mathbf{0} \\
\mathbf{k}_{1}^{\mathbf{b a}} & \mathbf{k}_{1}^{\mathbf{b b}} & \mathbf{0} & \mathbf{0} \\
\mathbf{0} & \mathbf{0} & \mathbf{0} & \mathbf{0} \\
\mathbf{0} & \mathbf{0} & \mathbf{0} & \mathbf{0}
\end{array}\right] .
$$

The structural tangent stiffness matrix of S2 can also be rewritten as

$$
\mathbf{K}_{2}\left(\sigma_{2}\right)=\left[\begin{array}{cccc}
\mathbf{k}_{2}^{\mathrm{aa}} & 0 & \mathbf{k}_{2}^{\mathrm{ac}} & \mathbf{k}_{2}^{\mathrm{ad}} \\
0 & 0 & 0 & 0 \\
\mathbf{k}_{2}^{\mathrm{ca}} & 0 & \mathbf{k}_{2}^{\mathrm{cc}} & \mathbf{k}_{2}^{\mathrm{cd}} \\
\mathbf{k}_{2}^{\mathrm{da}} & 0 & \mathbf{k}_{2}^{\mathrm{dc}} & \mathbf{k}_{2}^{\mathrm{dd}}
\end{array}\right] .
$$

Join the above two tangent stiffness matrices together, and the whole structure's tangent stiffness matrix can be obtained as follows:

$$
\left[\begin{array}{cccc}
\mathbf{k}_{1}^{\mathrm{aa}}+\mathbf{k}_{2}^{\mathrm{aa}} & \mathbf{k}_{1}^{\mathrm{ab}} & \mathbf{k}_{2}^{\mathrm{ac}} & \mathbf{k}_{2}^{\mathrm{ad}} \\
\mathbf{k}_{1}^{\mathbf{b a}} & \mathbf{k}_{1}^{\mathrm{bb}} & \mathbf{0} & \mathbf{0} \\
\mathbf{k}_{2}^{\mathrm{ca}} & \mathbf{0} & \mathbf{k}_{2}^{\mathrm{cc}} & \mathbf{k}_{2}^{\mathrm{cd}} \\
\mathbf{k}_{2}^{\mathrm{da}} & \mathbf{0} & \mathbf{k}_{2}^{\mathrm{dc}} & \mathbf{k}_{2}^{\mathrm{dd}}
\end{array}\right]
$$

Based on equation (11), the whole structure's nonlinear finite element equation at its reforming initial time $t_{0}$ can be written as 


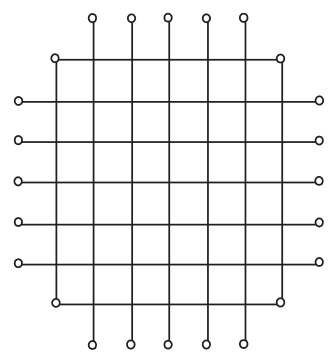

(a)

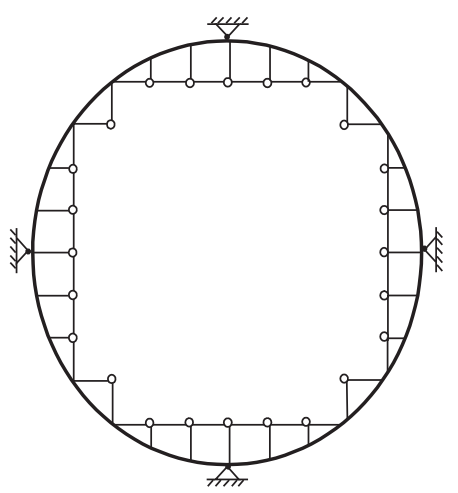

(b)

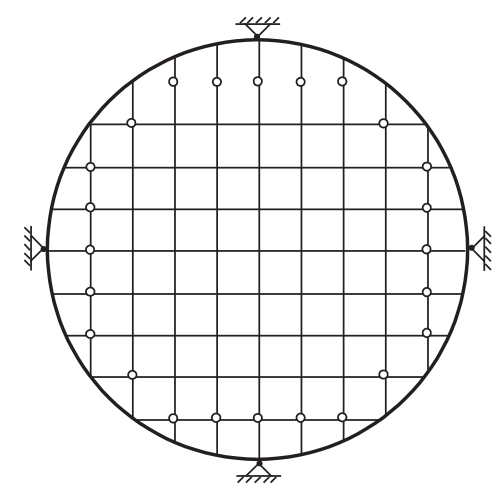

(c)

Figure 2: Reforming of cable-beam composite structure. (a) Inner cablenet structure released DOF constrains. (c) Reformed cable-beam composite structure. (b) Edge cable-beam structure released DOF constrains.

$$
\left[\begin{array}{cccc}
\mathbf{k}_{1}^{\mathrm{aa}}+\mathbf{k}_{2}^{\mathrm{aa}} & \mathbf{k}_{1}^{\mathrm{ab}} & \mathbf{k}_{2}^{\mathrm{ac}} & \mathbf{k}_{2}^{\mathrm{ad}} \\
\mathbf{k}_{1}^{\mathrm{ba}} & \mathbf{k}_{1}^{\mathbf{b b}} & \mathbf{0} & \mathbf{0} \\
\mathbf{k}_{2}^{\mathrm{ca}} & \mathbf{0} & \mathbf{k}_{2}^{\mathrm{cc}} & \mathbf{k}_{2}^{\mathrm{cd}} \\
\mathbf{k}_{2}^{\mathrm{da}} & \mathbf{0} & \mathbf{k}_{2}^{\mathrm{dc}} & \mathbf{k}_{2}^{\mathrm{dd}}
\end{array}\right]\left[\begin{array}{c}
\Delta \mathbf{u}^{\mathrm{a}} \\
\Delta \mathbf{u}^{\mathbf{b}} \\
\Delta \mathbf{u}^{\mathrm{c}} \\
\Delta \mathbf{u}^{\mathbf{d}}
\end{array}\right]=\left[\begin{array}{c}
\mathbf{F}_{1}^{\mathrm{a}}+\mathbf{F}_{2}^{\mathrm{a}}+\mathbf{R}_{1}^{\mathrm{a}}+\mathbf{R}_{2}^{\mathrm{a}} \\
\mathbf{R}_{1}^{\mathbf{b}} \\
\mathbf{R}_{2}^{\mathrm{c}} \\
\mathbf{R}_{2}^{\mathbf{d}}
\end{array}\right]
$$

In equation (12), $\mathbf{F}_{1}^{\mathbf{a}}$ and $\mathbf{F}_{2}^{\mathbf{a}}$ are cable pretensions' resultant forces of S1 and S2 at node-set $\boldsymbol{a}$.

When adding fixed nodes' DOF constraints and plugging initial values of $\mathbf{R}_{1}^{\mathbf{a}}, \mathbf{R}_{1}^{\mathbf{b}}, \mathbf{R}_{2}^{\mathbf{a}}, \mathbf{R}_{2}^{\mathbf{c}}$, and $\mathbf{R}_{2}^{\mathbf{d}}$ at time $t_{0}$ into equation (12), it can be further rewritten as

$$
\left[\begin{array}{cccc}
\mathbf{k}_{1}^{\mathbf{a a}}+\mathbf{k}_{2}^{\mathbf{a a}} & \mathbf{k}_{1}^{\mathrm{ab}} & \mathbf{0} & \mathbf{k}_{2}^{\mathbf{a d}} \\
\mathbf{k}_{1}^{\mathbf{b a}} & \mathbf{k}_{1}^{\mathbf{b b}} & \mathbf{0} & \mathbf{0} \\
\mathbf{0} & \mathbf{0} & \mathbf{1} & \mathbf{0} \\
\mathbf{k}_{2}^{\mathrm{da}} & \mathbf{0} & \mathbf{0} & \mathbf{k}_{2}^{\mathrm{dd}}
\end{array}\right]\left[\begin{array}{c}
\Delta \mathbf{u}^{\mathbf{a}} \\
\Delta \mathbf{u}^{\mathbf{b}} \\
\mathbf{0} \\
\Delta \mathbf{u}^{\mathbf{d}}
\end{array}\right]=\left[\begin{array}{c}
\mathbf{F}_{1}^{\mathbf{a}}+\mathbf{F}_{2}^{\mathbf{a}} \\
\mathbf{0} \\
\mathbf{0} \\
\mathbf{0}
\end{array}\right]
$$

According to equation (13), if the condition can be realized as follows:

$$
F_{1}^{a}+F_{2}^{a}=0
$$

Then the relations below can be gotten:

$$
\begin{gathered}
\left\{\begin{array}{l}
\Delta \mathbf{u}^{\mathbf{a}}=\mathbf{0}, \\
\Delta \mathbf{u}^{\mathbf{b}}=\mathbf{0}, \\
\Delta \mathbf{u}^{\mathbf{d}}=\mathbf{0},
\end{array}\right. \\
\left\{\begin{array}{l}
\boldsymbol{\sigma}_{1}^{\prime}=\sigma_{1}, \\
\boldsymbol{\sigma}_{2}^{\prime}=\sigma_{2} .
\end{array}\right.
\end{gathered}
$$

In equation (15), $\sigma_{1}^{\prime}$ and $\sigma_{2}^{\prime}$ are new prestresses of the reformed whole structure in its final balance state.

The above derivation shows that when equation (14) is satisfied, the reformed cable-beam composite structure can be in its balance state at time $t_{0}$, so nodes in node-sets $\mathbf{a}, \mathbf{b}$, and $\mathbf{d}$ will keep their original positions in structures $\mathrm{S} 1$ and S2, and every cable's pretension will also keep ideal value the same as S1 and S2.

What needs to be noted is the fact that when equation (14) cannot be strictly satisfied, if there is just the relation

$$
F_{1}^{a}+F_{2}^{a} \approx 0
$$

and if every element's deformations are just elastic, the final reformed structure in balance state will also have approximate equations as follows:

$$
\begin{gathered}
\left\{\begin{array}{l}
\Delta \mathbf{u}^{\mathbf{a}} \approx 0, \\
\Delta \mathbf{u}^{\mathbf{b}} \approx 0, \\
\Delta \mathbf{u}^{\mathbf{d}} \approx 0,
\end{array}\right. \\
\left\{\begin{array}{l}
\boldsymbol{\sigma}_{1}^{\prime} \approx \boldsymbol{\sigma}_{1}, \\
\boldsymbol{\sigma}_{1}^{\prime} \approx \boldsymbol{\sigma}_{2} .
\end{array}\right.
\end{gathered}
$$

This shows that if the resultant forces at node-set a can approximately be equal to 0 after reassembling structures $S 1$ and S2, the reformed cable-beam composite structure in its final balance state can almost keep its ideal state at initial time $t_{0}$.

Based on the above theoretical derivation, detailed calculation steps for the divided cable-beam composite structure can be given as follows. Firstly, for inner cablenet structure, add DOF constraints to the connection joints between S1 and S2, and calculate inner cablenet structure's optimal pretension distribution in condition with predetermined topologies and shapes. Secondly, for edge cable-beam structure, also add DOF constraints to the connection joints between S1 and S2, and iteratively adjust edge cables' pretensions to make equation (16) satisfied. When these two parts are joined together, the reformed cable-beam composite structure will keep its ideal state.

In iterative calculation process of pretension adjustment for edge cable-beam structure, cost of nonlinear finite element computation for equation (8) will be much less than that for the whole cable-beam composite structure, and so does the cost of pretension adjustment computation based on equation (8). Especially, for large cable-beam composite structure with complex topology and a huge number of cable elements, the above division method will have much better computational efficiency and convergence property.

\section{Pretension Optimization for Cablenet}

3.1. Pretension Optimization for Inner Cablenet. For the analysis on inner cablenet with predetermined topology, the 
main aim is to obtain a set of optimal cable pretensions that can make inner cablenet keep its ideal shape. Considering that balance matrix analysis method can efficiently analyze cablenet structure in balance state and that its solving process is just a list of linear calculations for equilibrium force equations, this method is used here to optimize inner cablenet's pretension distribution.

For cablenet structure with predetermined topology, when cable elements' gravity and sag are ignored, its structural balance state equilibrium equations of all unconstrained nodes can be established based on relations among nodal forces, and these equations can also be written as matrices below:

$$
\mathbf{A} \mathbf{T}=\mathbf{F} .
$$

In equation (18), $\mathbf{A}$ is cablenet's balance matrix, its rows number is equal to the total DOF number of unconstrained nodes, and its columns number is equal to the total number of cable elements. $\mathbf{T}=\left\{\begin{array}{llll}T_{1} & T_{2} & \ldots & T_{N}\end{array}\right\}^{\mathrm{T}}$, it is pretension column vector of cable elements, and $N$ is the total number of cable elements. $\mathbf{F}=\left\{\begin{array}{lllll}F_{1} & F_{2} & \ldots & F_{M}\end{array}\right\}^{\mathrm{T}}$, it is external load vector of unconstrained nodes, and $M$ is the total DOF number of unconstrained nodes.

Assume that the cablenet structure has no external loads; and when it is in balance state, its structural equilibrium force equations of all unconstrained nodes can be rewritten as

$$
\mathbf{A} \mathbf{T}=\mathbf{0} .
$$

Because there are no external loads, the right part of equation (19) is zero. This means that equation (19) is a homogeneous system of linear equations with balance matrix $\mathbf{A}$ as known quantity and pretension column vector $\mathbf{T}$ as unknown quantity. The form of the solution of equation (19) is dependent upon the form of coefficient matrix $\mathbf{A}$ [10-12].

When cable-beam composite structure has complex structure and large cable elements number, there are also large numbers of general solution's coefficients calculated from matrix A. So, in order to obtain cablenet's best pretension distribution, it is necessary to set these solution coefficients as variables and solve equation (19) with a reasonable objective function [9].

In order to make force evenly transfer in inner cablenet system, pretension distribution's evenness is set as objective function, and optimization mathematical model for inner cablenet can be established as follows.

$$
\begin{aligned}
\text { Find } \mathbf{X} & =\left(k_{1}, k_{2}, \ldots, k_{w}\right)^{T}, \\
\operatorname{Min} f(\mathbf{X}) & =\sum_{i=1}^{n}\left(T_{i}-T^{a}\right)^{2}, \\
\text { s.t. } \mathbf{A} \mathbf{T} & =0, \\
g(\mathbf{X}) & =T^{a}=\frac{\sum_{i=1}^{n} T_{i}}{n} \geq \mathrm{T}_{\mathrm{L}}^{a}, \\
T_{i} & >0, \\
X_{L} & \leq \mathbf{X} \leq X_{U} .
\end{aligned}
$$

In the above formulas, $\mathbf{X}$ is pretension solution coefficient variables and its number is $w$. Objective function $f(\mathbf{X})$ is the sum of the square of deviations between cable pretensions and their average pretension; this function can describe the evenness of cable pretensions. $T_{i}$ is the $i$ th cable element's pretension, $T^{a}$ is the mean of all cable pretensions, and $n$ is the number of cable elements. $\mathrm{T}_{\mathrm{L}}^{a}$ in constraints is the lower limit of $T^{a} ; X_{U}$ and $X_{L}$ are upper limit and lower limit of variables.

What we need to note is that only after the pretensions have been applied to cablenet structures, can they have determinate shapes and rigidities. In order to make sure cablenet structure has enough rigidity, an average minimum pretension value of cablenet is always given in advance to obtain reasonable structure's rigidity that is enough to resist adverse effects of environmental loads like rain and snow or that of vibration damage. Also, for some kinds of cablenet structures such as spaceborne cablenet antenna, because drive energy is restricted in space environment, antenna's motor cannot provide too large driving forces for cablenet's deployment, and, in this case, the optimal design for this type of cablenet structure often needs an average maximal pretension value to meet limits of driving forces. Of course, the values of $\mathrm{T}_{\mathrm{L}}^{a}, X_{U}$, and $X_{L}$ need to be determined on the basis of specific practical engineering conditions.

By solving the above optimization mathematical model, a set of optimal pretension solution coefficients under constraint conditions can be obtained, and, based on them, the best pretensions distribution of inner cablenet can finally be calculated.

Also, because balance matrix analysis method is based on linear force method and it ignores the relations between cablenet's geometry and material constitutive model in its analyzing process, it is necessary after using this method to further calculate all cables' original lengths by their pretension values and material constitutive relation.

\subsection{Iterative Pretension Calculatiuon Method for Edge Cable-} Beam System. Balance matrix method can efficiently analyze cablenet structure, but, for edge cable-beam system, if plugging cable pretensions calculated by balance matrix method into this type of divided structure, its beam elements will deviate their predetermined positions under the influence of cable pretensions. Also, cable elements' inner forces will decrease due to beam elements' deformations, which will lead to rigidity decrease and shape changing of the whole cablenet. So, balance matrix method cannot make sure cable-beam structure has the predetermined ideal state.

In order to reasonably combine cablenet's force equilibrium equation (14) with cablenet's shape adjustment under the influence of beam elements' deformations and make the whole cable-beam composite structure have predetermined ideal shape and even pretension distribution, we will join balance matrix method and nonlinear finite element method to iteratively analyze edge cable-beam structure. 
The specific measures are as follows. Firstly, use balance matrix method to solve equation (14) to calculate reasonable pretension values for edge cablenet in its ideal shape state; then plug these values as initial pretensions into edge cablebeam structure to make nonlinear finite element analyses. Next, calculate edge cablenet's pretension losses caused by beams' deformation and compensate them to make edge cablenet pretensions approximately revert to their initial values, which can ensure that cablenet has its predetermined rigidity. Then, update edge cablenet's shape according to beams' deformations, and again solve equation (14) to calculate initial pretension values for edge cablenet. Finally, repeat pretension losses compensation and initial pretensions updating for edge cablenet by using nonlinear finite element method and balance matrix method, respectively, till iteration convergence requirements are met.

In order to better describe the iterative calculation method, we use a simple two-dimensional cable-beam structure shown in Figure 3 as an example to reduce the complexity of problems. Black circular outside frame in Figure 3 is the composite structure's beam, point $G$ is the fixed node, and point $C$ and point $D$ are cable nodes with requirement for constant positions. Because these two nodes cannot deviate from their predetermined positions, cable $\mathrm{CD}$ between them is classified as inner cablenet, and the other cables AC, BC, DE, and DF between inner cablenet and beams are all edge cablenets. In order to highlight the adjustment function of edge cablenet, all edge cables are represented by spring shape in Figure 3. When pretensions are applied to cablenet, deformations of the whole cablebeam structure will occur, and the cablenet will deviate from its predetermined shape also. In this case, by adjusting edge cables' pretensions (they are, in effect, edge cables' lengths), inner cablenet can revert to its ideal shape.

Based on the above research approach, the iterative adjustment calculations for edge cable-beam structure can be summed up in five computational steps below.

The first step of this iterative analysis method is to calculate the optimal pretension distribution of edge cablenet in condition with its ideal shape state.

As shown in Figure 4, for edge cablenet, all joints connecting edge cable elements with beam elements are set to fixed nodes, and all joints connecting edge cable elements with inner cable elements are set to unconstrained nodes. Then edge cablenet's equilibrium force equations of all unconstrained nodes in their predetermined positions can be established:

$$
\overline{\mathbf{A}} \overline{\mathbf{T}}=-\mathrm{F}_{\text {in }} .
$$

In equation (21), $\overline{\mathbf{A}}$ is edge cablenet's balance matrix, its rows number is equal to the total DOF number of edge cablenet's unconstrained nodes, and its columns number is equal to the total number of edge cable elements. $\overline{\mathbf{T}}$ is pretension column vector of edge cable elements. $\mathbf{F}_{i n}$ is resultant pretension vector of inner cablenet on edge cable nodes that connect with inner cablenet.

If the number of general solution coefficients of equation (21) is larger than 1 , it is also necessary to calculate edge cablenet's best pretension distribution by using optimization

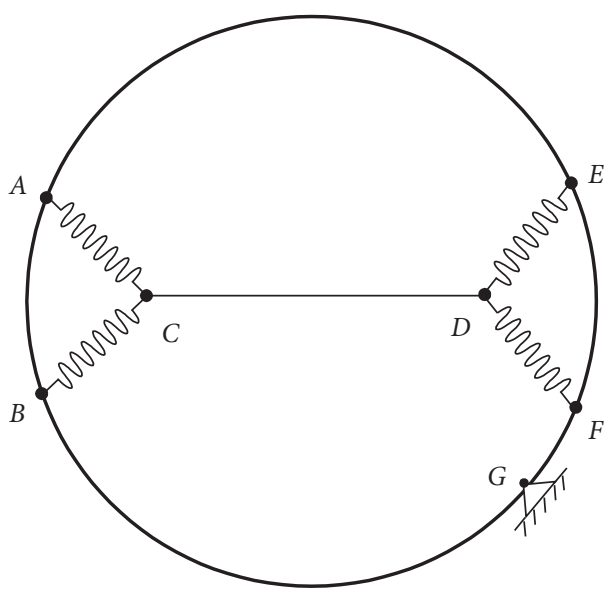

Figure 3: A simple two-dimensional cable-beam structure.

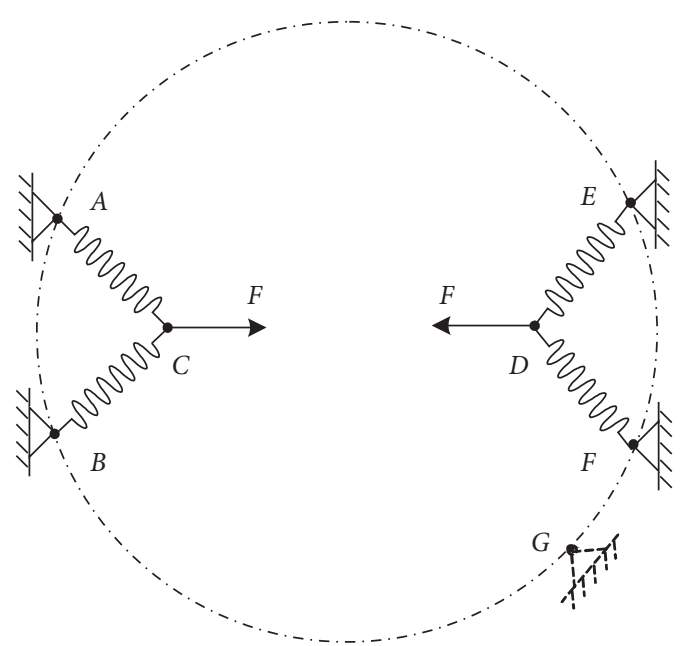

FIGURE 4: Edge cablenet's equilibrium force model in its ideal shape state.

method. This process is the same as the optimizing process for inner cablenet, so it will not be described in detail again.

The second step is the calculation of pretension losses of edge cables under the influence of beams' deformations.

When setting edge cablenet's pretensions calculated by the first step as initial pretensions and plugging them into edge cable-beam structure to make nonlinear finite element analyses, the beams in edge cable-beam structure will deform under the influence of edge cable pretensions, and, also, edge cables' pretensions will be weakened as beams deform (as shown in Figure 5). In this case, equation (14) will become false because of edge cable pretensions' changing. In order to make equation (14) be satisfied again, it is needed to iteratively adjust edge cable pretensions under the influence of beam deformations and make edge cable pretensions revert to inner cablenet's equilibrium forces at their connection joints.

For edge cablenet's pretension adjustment calculation, reverse pretension superposition method is used in the second step and the third step to compensate edge cablenet's 


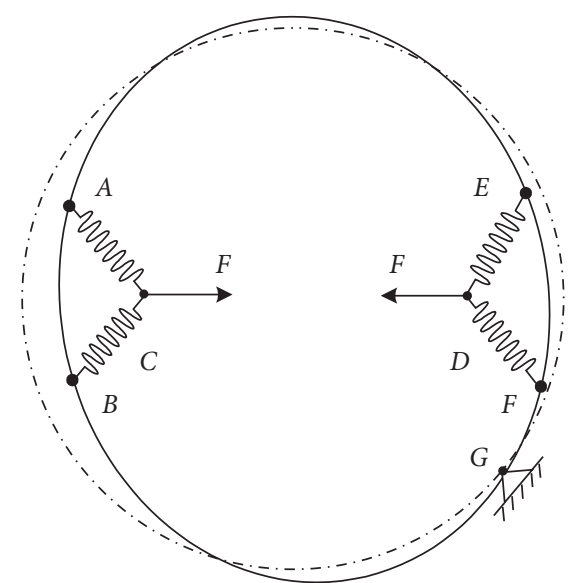

Figure 5: Deformed edge cable-beam structure.

pretension losses and make edge cable pretensions revert to their initial values calculated in the first step. The specific approaches are to first plug edge cable pretensions $\bar{T}$ calculated by balance matrix analysis method into nonlinear finite element model of edge cable-beam structure and make nonlinear finite element analyses. After the above calculation and through equation (22), edge cable elements' pretension losses $\Delta \overline{\mathbf{T}}^{*}$ under the influence of beam deformations can be obtained.

$$
\Delta \overline{\mathbf{T}}^{*(i)}=\overline{\mathbf{T}}-\overline{\mathbf{T}}^{*(i)} \text {. }
$$

In equation (22), $\overline{\mathbf{T}}$ are the edge cable pretensions calculated in the first step, $\overline{\mathbf{T}}^{*(i)}$ are the edge cable pretensions calculated by nonlinear finite element method in the $i$ th iterative adjustment step, and $\Delta \overline{\mathbf{T}}^{*(i)}$ are the edge cables' pretension losses in the $i$ th iterative adjustment step.

The third step is making edge cable pretensions revert to their predetermined level by iterative pretension losses compensation calculation.

In this step, firstly, it is needed to judge whether $\Delta \overline{\mathbf{T}}^{*}$ has met the preset demand for convergence precision. If the answer is yes, the edge cable pretensions calculated by nonlinear finite element method are considered approximately the same as the predetermined values; then the calculation can further go to the fourth step. If the answer is no, it is needed to compensate edge cables' pretension losses based on equation (23), and set $\overline{\mathbf{T}}+\Delta \overline{\mathbf{T}}^{*}$ as new edge cable pretensions' initial values, and then jump back to the second step to make nonlinear finite element analyses again.

$$
\overline{\mathbf{T}}^{(i+1)}=\overline{\mathbf{T}}^{(i)}+\Delta \overline{\mathbf{T}}^{*(i)} \text {. }
$$

In equation (23), $\overline{\mathbf{T}}^{(i+1)}$ are the edge cable pretensions' initial values in the $i$ th iterative step calculated by pretension losses compensation calculation. In the whole iterative calculation, the initial value $\overline{\mathbf{T}}^{(0)}$ is set as the preset edge cable pretensions for the first step.

When predetermined pretensions are applied to cablebeam composite structure, cables' tensile forces will cause beams' deformations, and, at the same time, pretensions of cablenet will decrease, which leads to relaxation and distortion of cablenet. In this case, we use edge cablenet pretension losses compensation method to make inner cablenet revert to its predetermined shape and rigidity. Because adjustment of edge cablenet pretension is actually achieved by changing edge cables' lengths, pretension losses compensation for edge cablenet is, in effect, the shortening or adjustment of edge cables, which can make inner cablenet retighten and revert to its original shape with predetermined pretension that ensures enough rigidity for the whole composite structure.

However, it is difficult to obtain edge cables' reasonable adjustment amounts only through one calculation step. So, we use a reverse compensation way to set $\overline{\mathbf{T}}+\Delta \overline{\mathbf{T}}^{*}$ as new edge cablenet's initial pretension and repeat this process to iteratively calculate the best edge cablenet's pretensions. The value of $\Delta \overline{\mathbf{T}}^{*}$ in this step is used to examine whether cablenet's pretensions have reverted to their predetermined levels. When $\Delta \overline{\mathbf{T}}^{*}$ is small enough to meet convergence requirement, we can say that the cablenets' pretensions in this step have reached predetermined levels, and the cablebeam composite structure has had predetermined rigidity.

The fourth step is updating edge cablenet's shape based on beams' deformations and judging whether the edge cablenet with new shape is adjustable. If the answer is yes, edge cable pretensions should be recalculated based on inner cablenet's pretensions in condition with edge cablenet's new shape.

The above iterative calculation can make edge cable pretensions revert to their predetermined level, but, at this moment, beam elements in edge cable-beam structure have also deformed under the influence of cable pretensions, and beams' deformations will also cause a shape change of edge cablenet. So, in this case, the edge cable pretensions calculated through the third step cannot finally become inner cablenet's equilibrium forces at the connection joints.

In order to solve this problem, it is necessary to update edge cablenet's shape based on beams' deformations, and recalculate edge cable pretensions that can match with inner cablenet's.

After iterative nonlinear finite element calculations in the third step, edge cablenet's new shape can be obtained based on position changes of joints that connect beam elements with edge cablenet. Next, edge cablenet's balance matrix in equation (21) can be further rewritten as $\left[\overline{\mathbf{A}}_{\text {new }}\right]$.

If the rank of matrix $\left[\overline{\mathbf{A}}_{\text {new }}\right]$ is not equal to the rank of equations' augmented matrix, equation (21) will have no solution, and, in this case, edge cable pretensions cannot be inner cablenet's equilibrium forces, and the adjustment calculations for edge cablenet have to stop and quit. Else, if the rank of matrix $\left[\overline{\mathbf{A}}_{\text {new }}\right]$ is equal to the rank of equations' augmented matrix, equation (21) will have solutions, and, next, it is needed to further judge whether the solutions are positive or negative based on the method in paper [13]. If all the solutions are positive, this means that all the edge cables can be tensional elements and the edge cablenet is adjustable in this case. Else, the solutions cannot be all positive; this means that some edge cables will be compressed or loose, and the edge cablenet is unadjustable in this case as well.

If the edge cablenet is unadjustable, the adjustment calculation for edge cablenet should quit, and equation (16) is considered to be satisfied in this case. Else, if the edge 
cablenet is adjustable, in order to make edge cable pretensions calculated by edge cablenet's new shape closer to their original best values, least square method [13] is used here to solve equation (21) to obtain a set of new edge cable pretensions $\overline{\mathbf{T}}_{\text {new }}$, the closest to its preset pretension $\overline{\mathbf{T}}$. After the above calculations, equation (24) can be further used to calculate the difference $\Delta \overline{\mathbf{T}}_{\text {new }}$ between $\overline{\mathbf{T}}_{\text {new }}$ and the edge cable pretensions in previous iteration step.

$$
\Delta \overline{\mathbf{T}}_{\text {new }}^{(i)}=\overline{\mathbf{T}}_{\text {new }}^{(i)}-\overline{\mathbf{T}}_{\text {new }}^{(i-1)} .
$$

In equation (24), $\Delta \overline{\mathbf{T}}_{\text {new }}^{(i)}$ is the difference of edge cablenet's preset pretensions calculated in the $i$ th iteration step, $\overline{\mathbf{T}}_{\text {new }}^{(i)}$ is the edge cablenet's preset pretensions calculated in the ith iteration step, and $\mathbf{T}_{\text {new }}(i)$ is the edge cablenet's preset pretensions calculated in the $i$-1 th iteration step.

The fifth step is judging whether the edge cablenet has the satisfied pretension distribution.

In this step, it is needed to judge whether $\Delta \overline{\mathbf{T}}_{\text {new }}$ has had enough convergence precision. If the requirement for convergence precision is met, the iteration is finished, and, at this moment, it is considered that the edge cable pretensions obtained in the current iteration step can ensure a stable match between beams' deformation and cablenet's shape. Else, if $\Delta \overline{\mathbf{T}}_{\text {new }}$ cannot meet the convergence precision requirement, $\Delta \overline{\mathbf{T}}_{\text {new }}$ will be set as edge cablenet's initial pretension values, and the calculation needs to skip to the second step to start the next iteration period again.

Figure 6 below is the calculation flow chart for analysis on cable-beam composite structure based on the above iteration steps.

In general, nonlinear finite element method can be used to analyze cable-beam composite structures, but crude cable pretensions can lead to larger nodal residual forces in nonlinear finite element iterative equation, which will make computational efficiency lower and even nonconvergence. Balance matrix analysis method can be used to obtain ideal pretensions for cablenet; when plugging these pretensions into cablenet's nonlinear finite element model, nodal residual forces are equal to 0 , so this method can ensure a high computational efficiency of nonlinear finite element calculation for complex cablenet structures. However, balance matrix analysis method cannot handle composite structures that consist of both cablenet and beams.

The iterative method in this section combines nonlinear finite element method with balance matrix analysis method and proposes innovative calculation steps to obtain the best cable pretensions that can ensure predetermined shape and rigidity for cable-beam composite structures. This method makes up for drawbacks of nonlinear finite element method and balance matrix analysis method, and its iterative calculation is not only able to analyze structures that consist of both cablenet and beams but also able to have a higher computational efficiency.

\section{An Example}

A kind of spaceborne deployable antenna made up of a $10 \mathrm{~m}$ cablenet system reflector and folding beams is chosen as an example in this paper. Its whole structure and divided structures are shown in Figures 7 and 8. The analysis for this cable-beam composite structure is aimed at making antenna's cablenet reflector keep its ideal preset shape and pretension distribution after antenna's deploying and pretension loading.

The antenna's inner cablenet system shown in Figure 8(a) consists of 170 cable nodes and 439 cable elements, and its edge cablenet system shown in Figure 8(d) consists of 132 cable nodes and 222 cable elements. In view of the structural symmetry of cablenet system, the inner cablenet system and edge cablenet system can be further divided into the same twelve subgroups shown in Figures $8(\mathrm{~b})$ and $8(\mathrm{e})$.

For complex cablenet structures, equation (19) will have a lot of pretension solution coefficients. Since too many coefficients will make optimization efficiency low, we should simplify balance matrix A according to cablenet's structural axial symmetry to reduce solution coefficients number.

For inner cablenet in Figure 6, based on its structural axial symmetry, it can be divided into the same twelve groups (as shown in Figure 6). For each group, pretension values of cable elements with the same axial symmetry locations are equal to each other.

After dividing inner cablenet into subgroups, equation (19) can be rewritten as

$$
\mathbf{A} \mathbf{E} \mathbf{T}^{\prime}=\mathbf{B} \mathbf{T}^{\prime}=0
$$

where $E$ is pretension transforming matrix, its row number is the total number of cable elements, and its column number is cable element number of grouped inner cablenets. $\mathrm{T}^{\prime}$ are pretension column vectors of cable elements of grouped inner cablenet. B is simplified structural balance matrix, its row number is the number of degrees of freedom of unrestrained nodes, and its column number is cable element number of grouped inner cablenets.

Because dimension of matrix $\mathbf{B}$ is much less than matrix A, the number of pretension solution coefficients will decrease a lot, which can make optimization for pretension solution coefficients much easier.

For grouped inner cablenet system, there are only 27 independent cable pretensions. After using balance matrix analysis method, the balance matrix of grouped inner cablenet structure has 330 rows, 27 columns, and 10 pretension solution coefficients. Then the optimization mathematical model for grouped inner cablenet can be established with these 10 coefficients as variables. In conditions with $T^{a} \geq 15 \mathrm{~N}$ and $T^{a} \geq 30 \mathrm{~N}$, the above optimization mathematical model is separately solved by using generalized reduced gradient method [14]. When upper limit of variables $X_{\mathrm{U}}$ is set to 1000 , lower limit of variables $X_{\mathrm{L}}$ is set to -1000 and variables' initial values are all set to 100 ; the optimal inner cablenets' pretension distribution obtained is shown in Figure 9.

Based on the calculated optimal pretensions of inner cablenet, balance matrix of grouped edge cablenet can be further established. The results of balance matrix analysis show that there are 12 independent edge cable pretensions and 4 pretension solution coefficients. Set these 4 coefficients 


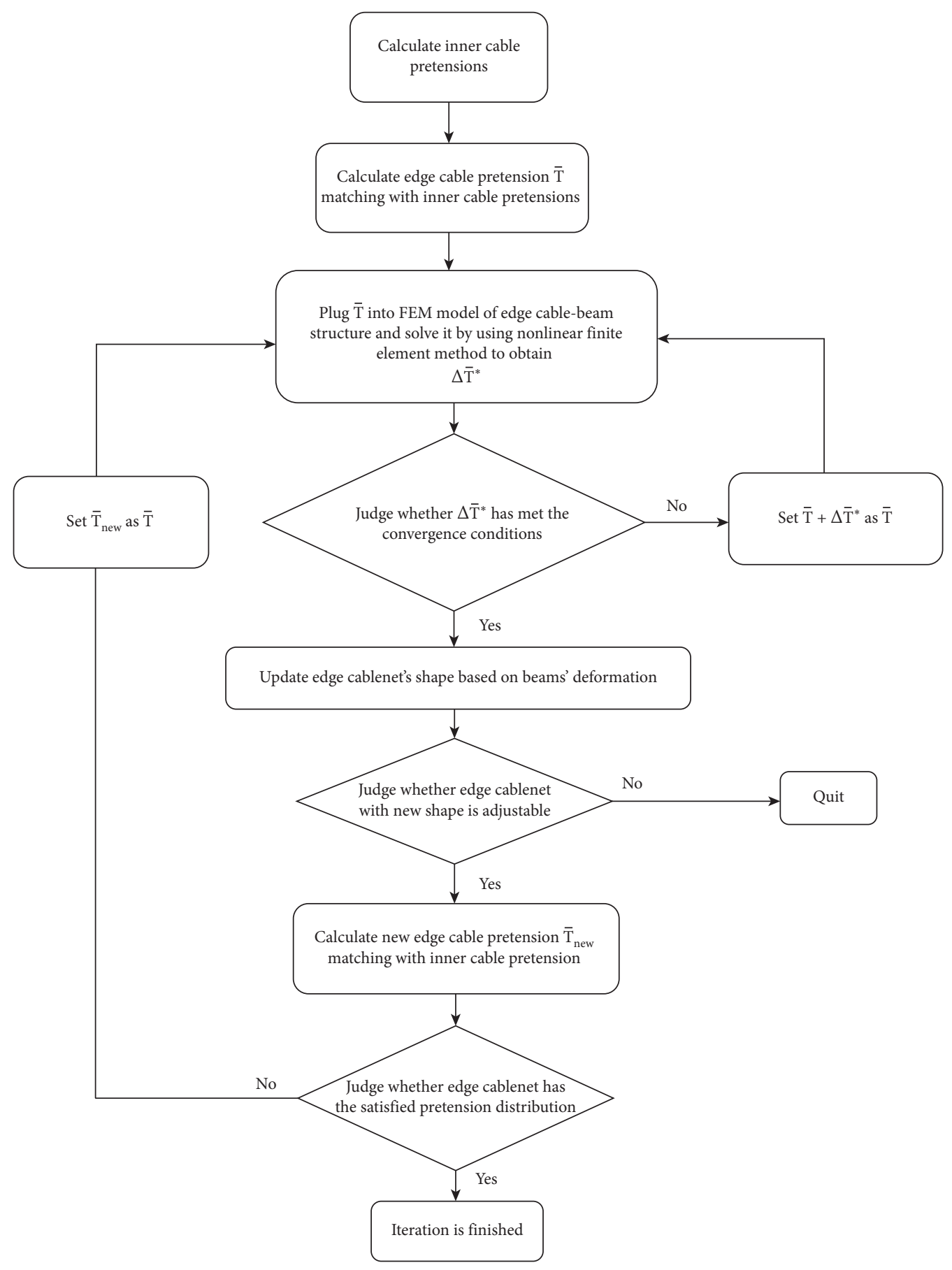

Figure 6: Calculation flow chart for analysis of cable-beam composite structure.

as variables, and calculate the grouped edge cablenet's optimization mathematical model in conditions with $T^{a} \geq 15 \mathrm{~N}$ and $T^{a} \geq 30 \mathrm{~N}$. When upper limit of variables $X_{\mathrm{U}}$ is set to 1000 , lower limit of variables $X_{\mathrm{L}}$ is set to -1000 , and variables' initial values are all set to 100 , the optimal edge cables' pretension distribution obtained is shown in Figure 10.

Next, iterative pretension losses compensation calculation method proposed in this paper is used for the above edge cable-beam composite antenna structure shown in Figure 8 to make antenna's cablenet reflector keep its ideal preset shape under influences of beams' deformations. The beams in antenna structure are circular carbon fiber tube with all outer diameters set to $10 \mathrm{~m}$, wall thicknesses set to $1 \mathrm{~mm}$, and modulus of elasticity set to $2.35 \mathrm{e} 11 \mathrm{~N} / \mathrm{m}^{2}$. All cables in antenna structure are circular aramid fiber ropes with all diameters set to $1 \mathrm{~mm}$ and modulus of elasticity set to $1.24 \mathrm{e} 11 \mathrm{~N} / \mathrm{m}^{2}$.

Put the optimal pretension values in condition with $T^{a} \geq 15 \mathrm{~N}$ into the edge cable-beam structure for iterative calculation, and when convergence precision is set to $0.05 \mathrm{~N}$, the root mean square value of edge cable pretension differences gets converged after 255 iterative steps. Then, set 


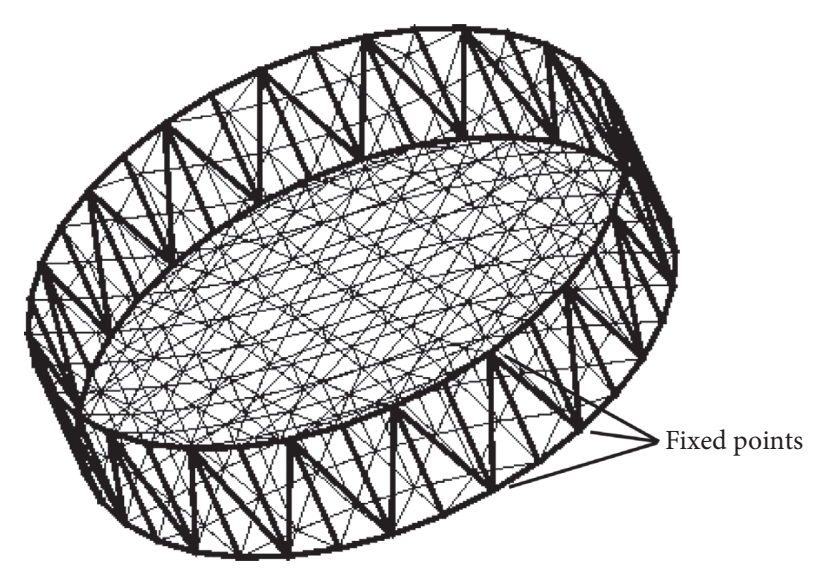

Figure 7: The whole structure of a kind of spaceborne cablenet deployable antenna.

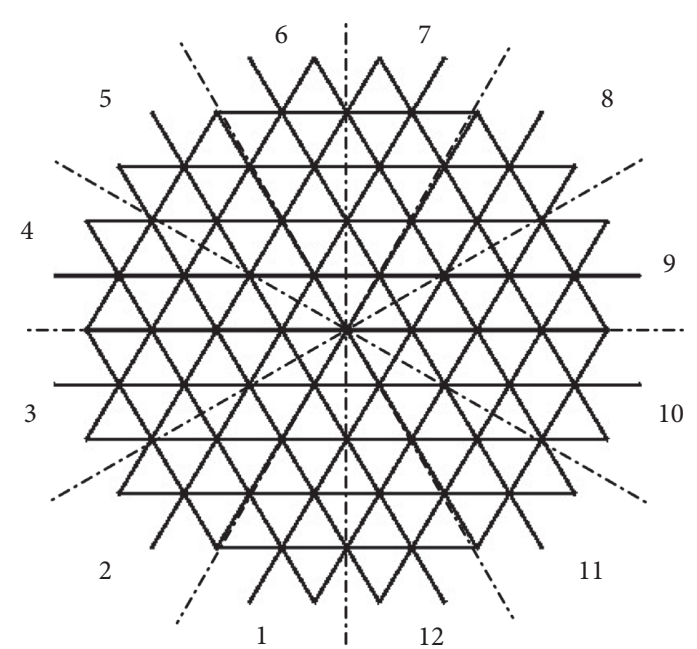

(a)

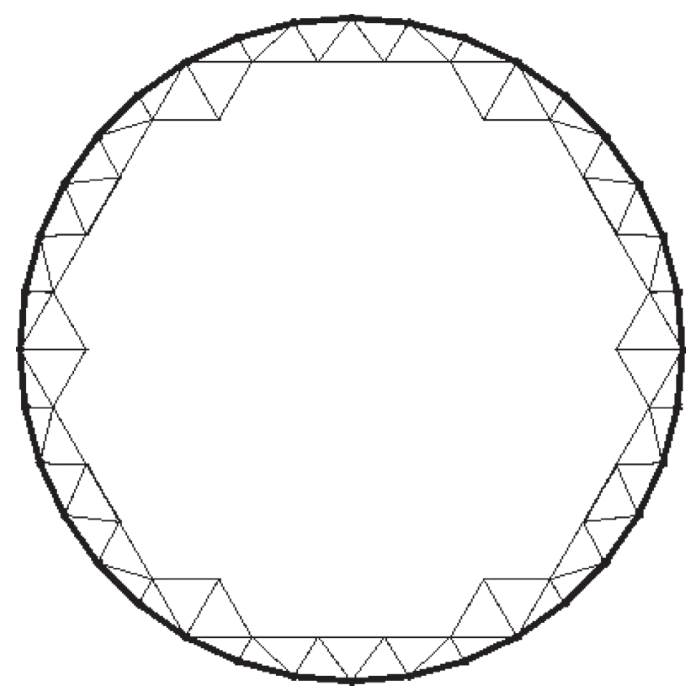

(c)

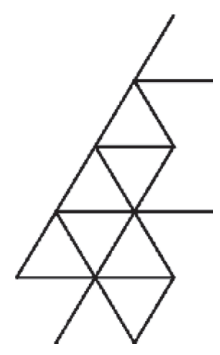

(b)

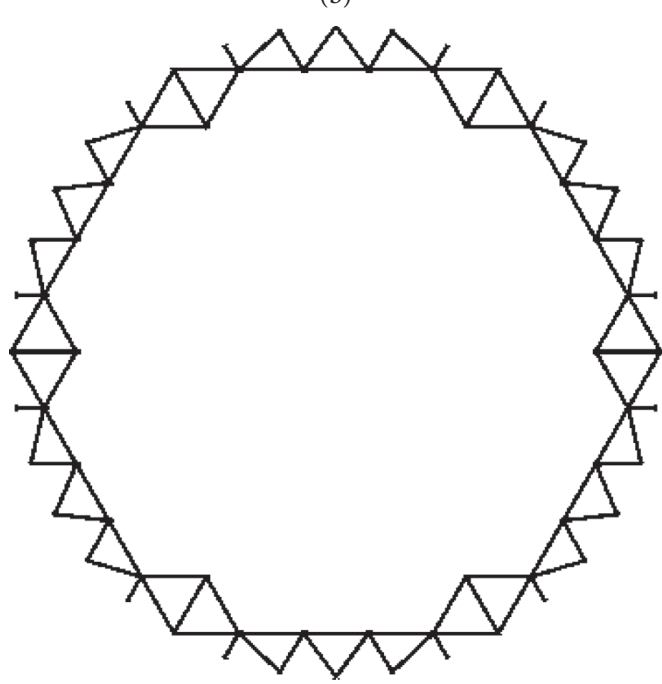

(d)

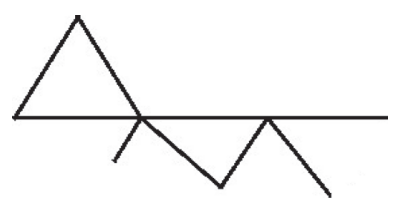

(e)

Figure 8: Divided structures of the spaceborne cablenet deployable antenna. (a) Inner cablenet system of the antenna. (b) Grouped inner cablenet system. (c) Edge cable-beam system of the antenna. (d) Edge cablenet system of the antenna. (e) Grouped edge cablenet system. 


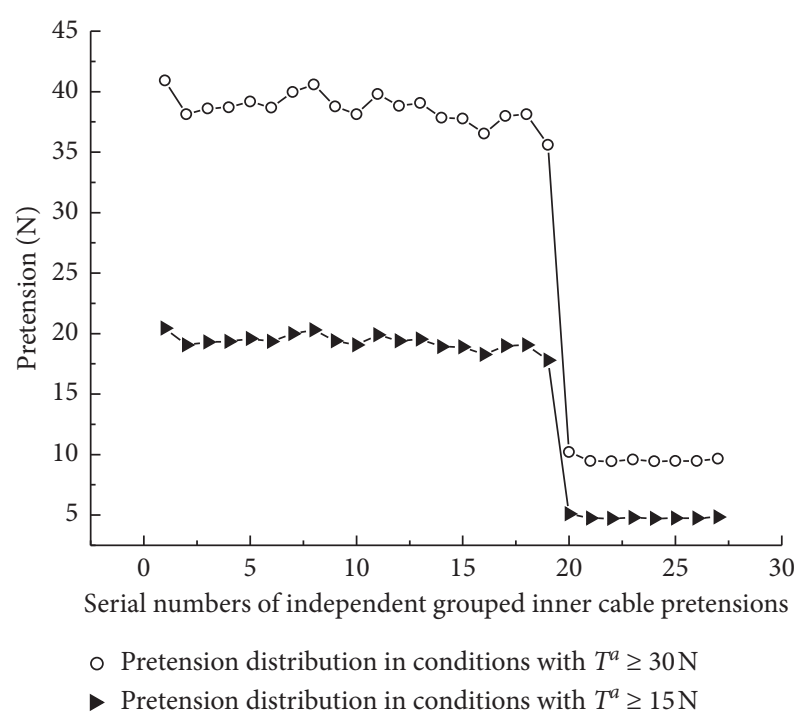

Figure 9: The optimal inner cables' pretension distribution in conditions with $T^{a} \geq 15 \mathrm{~N}$ and $T^{a} \geq 30 \mathrm{~N}$.

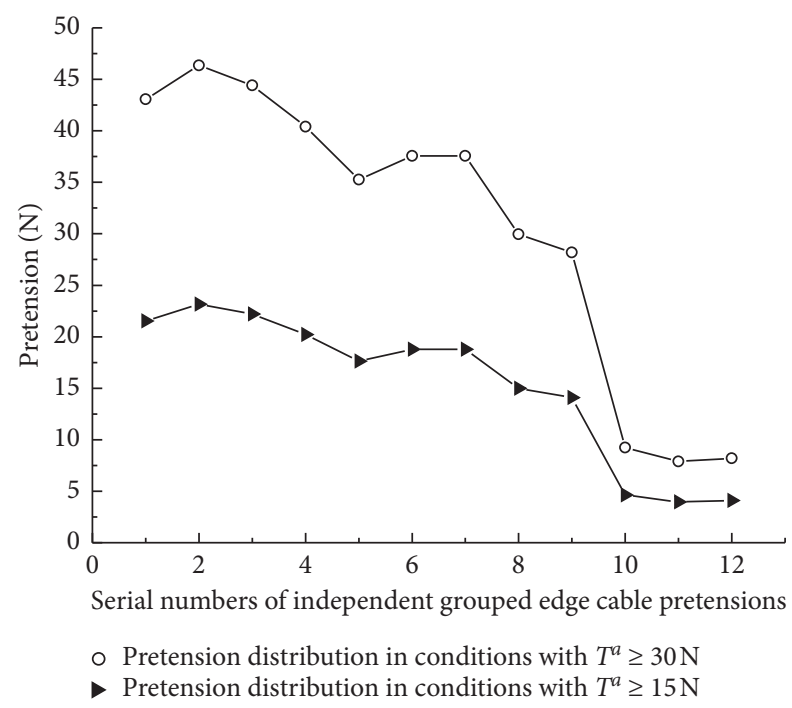

Figure 10: The optimal edge cables' pretension distribution.

inner cable pretensions to their optimal values in condition with $T^{a} \geq 15 \mathrm{~N}$, and separately put the optimal edge cable pretensions in condition with $T^{a} \geq 15 \mathrm{~N}$ and the iterative edge cable pretension results into antenna's structural model. After nonlinear finite element calculations, nodal displacement root mean square errors of inner cablenet are $0.1463 \mathrm{~mm}$ and $0.05706 \mathrm{~mm}$, the maximal nodal displacements are $0.2530 \mathrm{~mm}$ and $0.09454 \mathrm{~mm}$, and in these two different cases, the root mean square values of inner cable pretension differences are $1.1564 \mathrm{~N}$ and $0.04530 \mathrm{~N}$, and these values for edge cablenet are $2.9778 \mathrm{~N}$ and $0.1059 \mathrm{~N}$.

What needs to be additionally noted is the fact that when initial optimal cable pretensions are put into antenna's structural model to make nonlinear finite element calculations, these nonlinear calculations converge slowly because there are too many cable elements in the cablenet system. But when cable pretensions obtained from the above iterative compensation calculation are put into the same antenna's model, these nonlinear finite element calculations can converge much faster. This shows that iterative pretension results can make unbalanced nodal forces closer to 0 and shorten the time nonlinear calculations need, and this computational property also verifies the efficiency and validity of the iterative method proposed in this paper.

After the above iterative calculations, update edge cablenet's shape by position changes of connected nodes between edge cablenet and beams. Then, use the method in paper [13] to analyze edge cablenet's new equilibrium force equations; its results show these equations have general solutions, and all of them can be positive. Furthermore, least square method is used to calculate new edge cables' pretensions to make sure the solutions are the closest to their original values before beams' deformations. Its results show that the root mean square value of difference between them and edge cables' pretensions in last iterative step is $0.1374 \mathrm{~N}$. Because the convergence precision is set to $0.05 \mathrm{~N}$ and this root mean square value is larger than $0.05 \mathrm{~N}$, it is needed to continue to run the above iterative pretension losses compensation calculation steps again.

The iterative calculation converges after another shape updating step, and the final root mean square value of edge cablenet's pretensions is $0.03721 \mathrm{~N}$, and, in this case, it is clear that there has been a stable match between antenna's shape and cablenets' inner force distribution. In this converged state, the final nodal displacements' root mean square error of inner cablenet is $0.04142 \mathrm{~mm}$, the maximum nodal displacement is $0.07242 \mathrm{~mm}$, and the root mean square values of differences between inner cablenet's and edge cablenet's preset pretensions and these values in their balance state separately are $0.04075 \mathrm{~N}$ and $0.1862 \mathrm{~N}$.

Next, increase outer diameter of beam elements to $20 \mathrm{~mm}$ and make the above iterative calculations again. This time, the calculations converge after one edge cablenet shape updating step. The results of nodal displacements of inner cablenet and cable pretension errors in the whole iterative process are shown in Table 1. In this table, "Preset pretensions" represents the results calculated in conditions with putting preset pretensions obtained by

$T^{a} \geq 15 \mathrm{~N}$ into antenna's model, "The first iterative step" represents the results calculated in condition with putting pretensions obtained by the first iterative pretension losses compensation calculation into antenna's model, and "The second iterative step" represents the results calculated in condition with putting pretensions obtained by the second iterative calculation after edge cablenet shape updating into antenna's model.

At last, in conditions with beams' outer diameter equal to $20 \mathrm{~mm}$ and the average pretension of inner cablenet and edge cablenet greater than or equal to $30 \mathrm{~N}$, the iterative pretension losses compensation algorithm is run again. In this case, the calculations converge after two edge cablenet shape updating steps; the results of nodal displacements of inner cablenet and cable pretension errors in the whole iterative process are shown in Table 2. 
TABLE 1: Results of iterative pretension losses compensation calculation after increasing beam's outer diameter.

\begin{tabular}{lcccc}
\hline & $\begin{array}{c}\text { The root mean square error of } \\
\text { inner cablenet's nodal } \\
\text { displacements }(\mathrm{mm})\end{array}$ & $\begin{array}{c}\text { The maximum nodal } \\
\text { displacement of inner } \\
\text { cablenet }(\mathrm{mm})\end{array}$ & $\begin{array}{c}\text { The root mean square value } \\
\text { of inner cablenet's } \\
\text { pretension differences }(\mathrm{N})\end{array}$ & $\begin{array}{c}\text { The root mean square value } \\
\text { of edge cablenet's pretension } \\
\text { differences }(\mathrm{N})\end{array}$ \\
\hline $\begin{array}{l}\text { Preset } \\
\text { pretensions }\end{array}$ & 0.08872 & 0.1566 & 0.5648 & 1.3035 \\
$\begin{array}{l}\text { The first } \\
\text { iterative step }\end{array}$ \\
$\begin{array}{l}\text { The second } \\
\text { iterative step }\end{array}$
\end{tabular}

TABLE 2: Results of iterative pretension losses compensation calculation in condition with the average pretension of inner cablenet and edge cablenet greater than or equal to $30 \mathrm{~N}$.

\begin{tabular}{|c|c|c|c|c|}
\hline & $\begin{array}{l}\text { The root mean square error of } \\
\text { inner cablenet's nodal } \\
\text { displacements }(\mathrm{mm})\end{array}$ & $\begin{array}{l}\text { The maximum nodal } \\
\text { displacement of inner } \\
\text { cablenet }(\mathrm{mm})\end{array}$ & $\begin{array}{l}\text { The root mean square value } \\
\text { of inner cablenet's } \\
\text { pretension differences }(\mathrm{N})\end{array}$ & $\begin{array}{l}\text { The root mean square value } \\
\text { of edge cablenet's pretension } \\
\text { differences }(\mathrm{N})\end{array}$ \\
\hline $\begin{array}{l}\text { Preset } \\
\text { pretensions }\end{array}$ & 0.1822 & 0.3211 & 1.1313 & 2.6155 \\
\hline $\begin{array}{l}\text { The first } \\
\text { iterative step }\end{array}$ & 0.07414 & 0.1293 & 0.06577 & 0.1149 \\
\hline $\begin{array}{l}\text { The second } \\
\text { iterative step }\end{array}$ & 0.06786 & 0.1204 & 0.06438 & 0.1513 \\
\hline $\begin{array}{l}\text { The third } \\
\text { iterative step }\end{array}$ & 0.06743 & 0.1196 & 0.06431 & 0.1556 \\
\hline
\end{tabular}

The above three iterative calculations' results suggest that although the spaceborne deployable antenna in this example is a complex cable-beam composite structure with a large number of cable elements, when the edge cablenet's pretensions calculated by the iterative pretension losses compensation algorithm are put into antenna's structural model to make nonlinear finite element analyses, antenna's inner cablenet can revert to its preset ideal shape well, and, also, computational efficiency and convergence property of antenna's nonlinear finite calculations are greatly improved. All of these show that the method proposed in this paper is efficient and valid for complex cable-beam composite structure's nonlinear analysis.

\section{Conclusions}

In this paper, for analyzing complex cable-beam composite structures, they are first divided into inner cablenet system and edge cable-beam system; then an iterative calculation method combined with balance matrix analysis method and nonlinear finite element method is used to make an efficient analysis.

This iterative calculation method is used for a spaceborne deployable antenna example. Based on its computed results, four conclusions can be obtained as follows.

(1) The computed results of the example show the iterative pretension losses compensation calculation method proposed in this paper can make complex cable-beam composite structure's cablenet system keep its preset ideal shape in condition with pretension loads.

(2) For inner cablenet system divided from cable-beam composite structure, balance matrix analysis method is used to efficiently calculate its ideal pretension distribution. For the other edge cable-beam systems, nonlinear finite element method and balance matrix analysis method are combined together for iterative use for the calculation, and because edge cable-beam system is just a simpler structure with only a small number of cable elements, the common problems such as convergence difficulty and long computation time in nonlinear finite element calculation for complex cable-beam composite structure will not happen here. By handling special cases with special methods, the whole calculation efficiency is largely improved. Furthermore, because the iterative pretension losses compensation calculation for edge cablenet system includes the influences of beams' deformations, when the edge cable pretension results are put into the whole cable-beam composite structure model to make a nonlinear finite element calculation, the nodal unbalance forces will be closer to zero, which will also make the nonlinear finite element calculation for the whole cable-beam composite structure much easier to converge.

(3) The results of the example show that when beam's outer diameter is increased, displacement errors of cablenet system will decrease. In this case, edge cable-beam system's iterative pretension losses compensation calculation just needs fewer iterative steps to make antenna's cablenet system return its preset ideal shape, so this suggests that increasing beam elements' stiffness to some extent can make iterative pretension losses compensation calculation easier to converge. 
(4) For design on edge cablenet system, it is needed to make edge cablenet have enough pretension solution coefficients and an appropriate cable topology to ensure that edge cablenet's equilibrium force equation (21) has all positive pretension solutions under influence of beam deformations, and that will also be helpful for edge cablenet's iterative compensation calculation.

Furthermore, It is needed to note that, in order to highlight edge cablenet's significance in iterative calculation method, its preset pretensions are solved independently in this paper. But there are actually many other ways to calculate edge cablenet's ideal pretensions; for example, if using balance matrix analysis method to calculate the whole cablenet's pretension distribution, edge cablenet's pretension can be directly obtained according to its position in the whole cablenet. Obviously, this method is more efficient for the whole pretension calculation process.

\section{Data Availability}

The data used to support the findings of this study are available from the corresponding author upon request.

\section{Conflicts of Interest}

The author has no conflicts of interest to declare.

\section{Acknowledgments}

Financial supports by Xi'an Fanyi University research team fund (XFU17KYTDC01) are gratefully acknowledged.

\section{References}

[1] G. Tibert, Numerical Analyses of Cable Roof Structures, pp. 1-24, Royal Institute of Technology Department of Structural Engineering, Stockholm, Sweden, 1999.

[2] L. Ruiwei, G. Hongwei, L. Rongqiang et al., "Shape accuracy optimization for cable-rib tension deployable antenna structure with tensioned cables," Acta Astronautica, vol. 140, pp. 66-77, 2017.

[3] P. Jiang, Q. M. Wang, and Q. Zhao, "Optimization and analysis on cable net structure supporting the reflector of the large radio telescope fast," Applied Mechanics and Materials, vol. 94-96, pp. 979-982, 2011.

[4] G. Tibert, Depolyable Tensegrity Structure for Space Applications, pp. 32-64, Royal Institute of Technology Department of Mechanics, Stockholm, Sweden, 2002.

[5] G. Li, L. Shen, and Y. Luo, "Inverse iteration method in formfinding of cable structures," Building Structure, vol. 36, no. 4, pp. 74-76, 2006.

[6] C.-D. Li, J.-Q. Yi, Y. Yu, and D.-B. Zhao, "Inverse control of cable-driven parallel mechanism using type-2 fuzzy neural network," Acta Automatica Sinica, vol. 36, no. 3, pp. 459-464, 2010.

[7] Y. Tang, T. Li, and X. Ma, "Form finding of cable net reflector antennas considering creep and recovery behaviors," Journal of Spacecraft and Rockets, vol. 53, no. 4, pp. 610-618, 2016.
[8] S. Pellegrino, "Analysis of prestressed mechanisms," International Journal of Solids and Structures, vol. 26, no. 12, pp. 1329-1350, 1990.

[9] S. Pellegrino, "Structural computations with the singular value decomposition of the equilibrium matrix," International Journal of Solids and Structures, vol. 30, no. 21, pp. 3025-3035, 1993.

[10] M. Misawa and Y. Ohkami, "Stiffness design of deployable satellite antennas in deployed configuration," Journal of Spacecraft and Rockets, vol. 35, no. 3, pp. 380-386, 1998.

[11] C. A. Rogers, W. L. Stutzman, T. G. Campbell, and J. M. Hedgepeth, "Technology assessment and development of large deployable antennas," Journal of Aerospace Engineering, vol. 6, no. 1, pp. 34-54, 1993.

[12] M. C. Natori, H. Hirabayashi, N. Okuizumi et al., "A structure concept of high precision mesh antenna for space VLBI observation," in Proceedings of the 43rd AIAA/ASME/ASCE/ AHS/ASC Structures, Structural Dynamics, and Materials Conference, vol. 22-25, ASME, Denver, Colorado, USA, April 2002.

[13] H. Ohyama and S. Kawamata, A Problem of Surface Design for Prestressed Cable nets, McGraw-Hill, in Proceedings of the IASS Pacific Symposium-Part II, McGraw-Hill, Tokyo, Japan, pp. 17-23, October 1971.

[14] G. You and P. Yang, "Multiobjective shape optimization for deployment and adjustment properties of cable-net of deployable antenna," Mathematical Problems in Engineering, vol. 2015, p. 5, 2015. 